\title{
OPINION DYNAMICS IN COMPLEX NETWORKS
}

\author{
Wei Zhang ${ }^{\text {a }}$, Ming-sheng $\mathrm{He}^{\mathrm{b}}$, and Rui Jin ${ }^{\mathrm{b}}$ \\ ${ }^{a}$ School of Management, Harbin Institute of Technology, A10, 92 West Dazhi Street, Harbin, China \\ ${ }^{\mathrm{b}}$ School of Social Development, East China University of Political Science and Law, Shanghai, China \\ E-mail: zh-wei@outlook.com
}

Received 25 April 2013; revised 12 July 2013; accepted 4 December 2013

\begin{abstract}
Based on the modification of the convergence parameter $\mu$ in the Weisbuch-Deffuant (WD) model, we investigated the influence of the network structure on opinion dynamics by comparing the processes of opinion dynamics in the Watts-Strogatz (WS) small-world network and Barabási-Albert (BA) scale-free network. The simulation results present that the time evolution of opinions does not always end up with a consensus; the final number of opinion clusters depends on the value of the bounded confidence but compared with the situation in the original WD model the effect of the bounded confidence is different when dynamics happens in the small-world network and BA network. Furthermore, the structural cohesion of the network is strengthened by the rich nodes in the BA network, which make the opinions evolve at a much faster rate than those in the small-world network.
\end{abstract}

Keywords: opinion dynamics, complex network, structural cohesion, rich nodes, Weisbuch-Deffuant model

PACS: 87.23.Ge, 05.45.-a, 89.65.-s

\section{Introduction}

The opinion dynamics modelling, which is very popular in modern sociophysics researches, represents a challenging field where ideas from statistical physics and nonlinear science can be possibly applied to understand the emergence of collective behaviours, like consensus or polarization in social groups. According to the distribution of agents' opinions, opinion dynamics models can be divided into two categories. One category includes discrete models in which agents' opinion can only adopt a finite set of values, such as the Ising model [1], Sznajd model [2], and voter models. Taking the Sznajd model, for example, individuals have to be in one of the two states which are represented by +1 and -1 respectively at each time-step and change their own states by adopting that of their neighbours according to some simple sets. The other category includes continuous models in which the opinion of an agent is expressed as a real number in a finite interval such as $[0,1]$. The WeisbuchDeffuant (WD) model [3-5], and the HegselmannKrause (HK) model [6] are two typical cases of this category. In these two models, opinion exchanges can only take place when the difference between the two agents' opinions is below the confidence bound which can also be called the threshold $6-$ 8]. Though discrete models are useful to represent situations where binary choices are a good description of the problem, continuous models can be used more widely because people usually hold obscure attitudes towards a certain issue in the social world which is also the basic idea of fuzzy mathematics and fuzzy statistics.

In continuous opinion dynamics models, researchers usually suppose that opinion exchanges happen between two randomly chosen agents whose opinions are distributed between the range $[0,1]$ if their difference is smaller than the given 
threshold. This kind of settings could simplify the establishment of mathematical models and reduce the amount of calculation work, but some rules of the models are improper, for example, one agent can exchange his opinion with anyone else in the group. According to the ideas of the complex network theory, our society is a big network in which individuals are the nodes while the links between them are the edges. Information can only be transmitted through the edges between every two nodes in the network and proliferated across nearly the whole network by this means. Therefore, using the ideas coming from the complex network theory to modify opinion dynamics models and analyse the effect of network topology is drawing attention of the researches in this field. For example, Weisbuch et al. suppose that individuals are restricted to a square lattice and interaction could happen between close neighbours only [ 3 , 4 ]. However, their hypotheticals on the social networks are questioned by some other researchers [9, 10] because they may be applied better to trees in an orchard than to human beings [9]. As an improvement to the WD model, Balazs and Alain investigated the consensus formation on adaptive networks and found that the scenarios of transitions between consensus and polarized states are more robust on adaptive networks [11, 12]. Social connections with different structures such as the Erdős Rényi random graph, smallworld network, and scale-free network are drawn into the opinion dynamics modelling [13-17]. Though some of their conclusions remain open to question, their perspectives are worth considering. In fact, comparative analysis and some key properties (rich-club phenomenon [18-20], for example) of complex networks are seldom involved in current researches.

In this paper, we investigate the opinion dynamics in complex networks by modifying the convergence parameter $\mu$ in the WD model, so that it will be more in line with reality, and reveal the influence of the topological properties of the network by comparing the processes of opinion dynamics in the Watts-Strogatz (WS) small-world network and the Barabási-Albert (BA) scale-free network.

\section{Opinion dynamics model}

The WD model was first proposed by Weisbuch and Deffuant [3], and it is an agent-based model driven by repeated averaging under the bounded confidence. The model supposes that all the agents in the group have an initial distribution of opinions, and the value of one's opinion is limited in a finite interval $[0,1]$. In the WD model, individuals meet in random pairwise encounters in a given connectivity network at each time-step. They re-adjust their opinions when the difference of their opinions is smaller than the threshold. Because the statistics of the WD model is excellent [9], it has captured the interest of many researchers [21].

Consider a set of $N$ agents. Each agent is connected with others and has its own opinion which is represented by a real number between 0 and 1 . At the time-step $t$, opinions of two randomly chosen agents $i$ and $j$ are denoted by $x_{i}(t)$ and $x_{i}(t)$, respectively. The bounded confidence is usually denoted by $\varepsilon$. If $\left|x_{i}(t)-x_{i}(t)\right| \leq \varepsilon$, which means the difference between $i$ and $j$ is smaller than the given threshold, their opinions will be adjusted according to the following rules:

$$
\left\{\begin{array}{l}
x_{i}(t+1)=x_{i}(t)+\mu\left(x_{j}(t)-x_{i}(t)\right) \\
x_{j}(t+1)=x_{j}(t)+\mu\left(x_{i}(t)-x_{j}(t)\right) .
\end{array}\right.
$$

In the WD model, $\mu$ is the convergence parameter whose value is normally restricted to the interval $[0,0.5]$. The value of $\mu$ can affect the convergence time of all the opinions as well as the number of final opinion groups. When $\mu$ is small, individuals slightly change their opinions during the meeting; while in an opposite situation, individuals may change their opinions mostly. In most studies, $\mu$ is usually given the value 0.5 [22], and the dynamic rules become

$$
\left\{\begin{array}{l}
x_{i}(t+1)=x_{i}(t)+0.5\left(x_{j}(t)-x_{i}(t)\right) \\
x_{j}(t+1)=x_{j}(t)+0.5\left(x_{i}(t)-x_{j}(t)\right)
\end{array}\left|x_{i}(t)-x_{j}(t)\right| \leq \varepsilon .\right.
$$

As is represented in the WD model, opinions of two randomly chosen agents will be the same after opinion exchange. In other words, interacting agents can always reach an agreement at any time-step. Actually, the situation where both agents hold the same opinion after their interbehaviour could happen only if the interbehaviour is deep enough. Because of numerous participants in the spreading process of public opinion and the heterogeneity of individuals, the 
deep interbehaviour of each selected couple rarely happens. Therefore, the values of $\mu$ will be different for different agents, and it indicates how likely one agent could accept the perspective of another agent. For that matter, some researchers improve the model by assigning $\mu$ with a different value during the interacting process from the other's view, and they regard $\mu$ as a function of the degree of an agent with which one interacts [23, 24]. In this paper, we adopt another strategy to make $\mu$ be more in line with reality from the agent's own point of view. We set that the value of $\mu$ could be higher than 0.5 , which is different from the classical WD model because one's opinion could be changed significantly by other agents if he/ she is not confident. We suppose the value of $\mu$ obeys a certain type of statistical distribution, such as random distribution and normal distribution. With $\mu$ increasing, one's acceptance of others' opinions will be enhanced. In this case, the new modes of opinion dynamics can be represented as follows:

$$
\begin{aligned}
& \text { if }\left|x_{i}(t)-x_{j}(t)\right| \leq \varepsilon,\left\{\begin{array}{l}
x_{i}(t+1)=x_{i}(t)+\mu_{i}\left(x_{j}(t)-x_{i}(t)\right) \\
x_{j}(t+1)=x_{j}(t)+\mu_{j}\left(x_{i}(t)-x_{j}(t)\right)
\end{array}\right. \\
& \text { otherwise, }\left\{\begin{array}{l}
x_{i}(t+1)=x_{i}(t) \\
x_{j}(t+1)=x_{j}(t)
\end{array}\right.
\end{aligned}
$$

where $i$ and $j$ are not randomly chosen from the group. As two nodes of the network, an edge between them is the basis of their opinion exchanges.
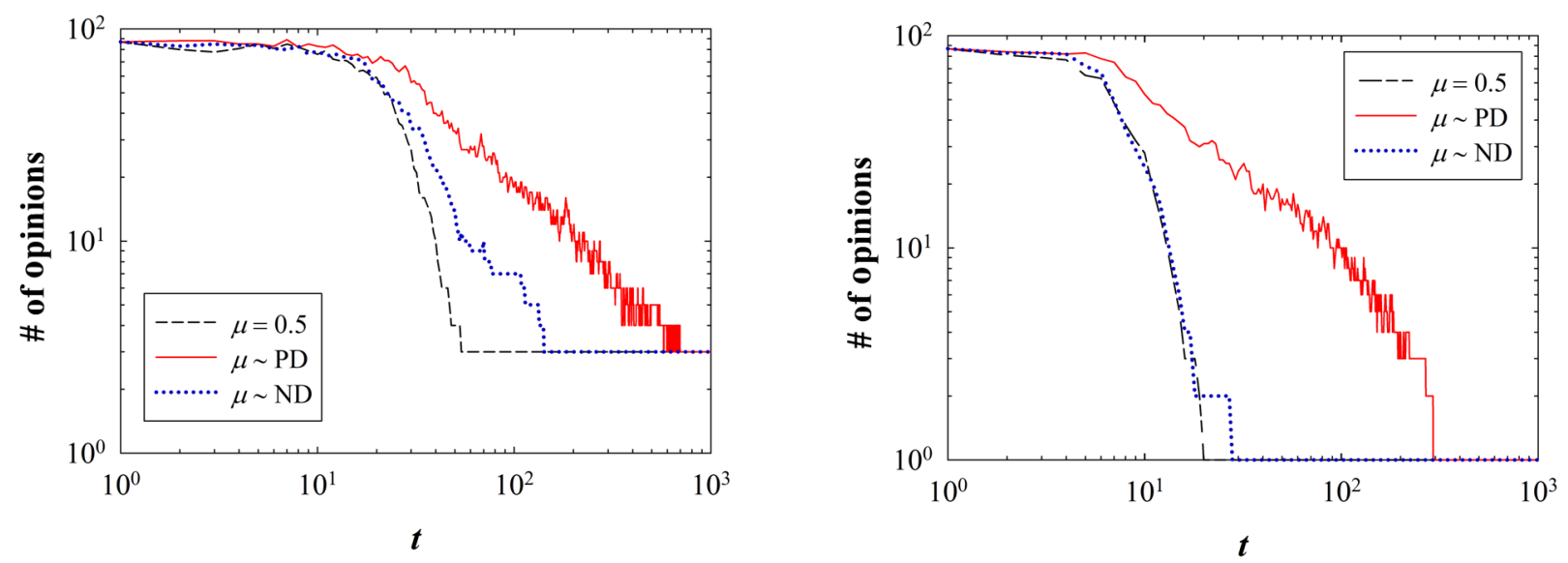

Fig. 1. Fluctuations of opinions' number with the convergence parameter $\mu$ equalling 0.5 and obeying random distribution (denoted by $\mu \sim \mathrm{RD}$ ) and normal distribution (denoted by $\mu \sim \mathrm{ND}$ ). The bounded confidence $\varepsilon$ in the two figures equals 0.2 (left) and 0.5 (right), respectively. Note that all the values have retained two digits after the decimal point for the convenience of calculating, so the maximum of the total number of opinions is $10^{2}$.

As an important parameter in the WD model, the value of $\mu$ determines the convergence time between individuals. We have suggested that its value ranges from 0 to 1 ; with $\mu$ increasing, the variation amplitude of one's opinion changing will be strengthened. As expounded in Section 2, the convergence parameter $\mu$ is heterogeneous for the agents involved in the opinion evolution process. In order to investigate the influence of $\mu$ 's distribution on opinion dynamics, we analyse the model with it following two classical statistical distributions: random distribution (RD) and normal distribution (ND), and compare the simulation results to the case where $\mu$ is fixed and equals 0.5 as well.

Set that the total number of agents $N=200$, iterations $T=1000$, and the initial opinion of agent varies continuously from 0 to 1 randomly. We carry out simulations with different values of $\varepsilon$ as shown in Fig. 1.

The results in Fig. 1 show the effects of the convergence parameter $\mu$ on the diffusion of opinions.

In the network of opinion dynamics, one can only ing to him, so $j$ is randomly chosen from the set of agents who connect to $i$ directly.

\section{Influence of convergence parameter $\mu$ on}


It can be found that the final number of opinions equals 3 when $\varepsilon=0.2$ and equals 1 when $\varepsilon=0.5$. From this we can suppose that the final number of opinions will not change with the distribution of $\mu$. However, it will affect the rate of opinion dynamics significantly. In this regard, individuals' opinions are converging at the fastest pace when $\mu$ is given a fixed value 0.5 , followed by $\mu$ obeying normal distribution with the average value equalling 0.5 , and the pace is the slowest when the value of $\mu$ is randomly distributed. Therefore, it makes sense for us to take the distribution of the convergence parameter $\mu$ into account in modelling opinion dynamics. During the practice, if we can only obtain the limited knowledge about the distributions of individuals, such as the mean value or variance, it is relatively a better choice to use normal distribution to simulate real distribution according to the maximum entropy theory [25]. Generally, most individuals in our real society are eclectic; donkeys, whose opinions are hard to be affected by others, and copycats, who always follow the opinion of others to a large extent, are few in numbers. Therefore, it is better to let $\mu$ obey normal distribution in the following simulations.

\section{Influence of network structure on opinion dynamics}

\subsection{Opinion dynamics in small-world network}

Small-world networks are the networks having both a small value of the average shortest path length like random graphs and a high clustering coefficient like regular lattices, and they have been introduced as an interpolation between ordered and random graphs to capture two specific features of real neural, social, and technological networks [26]. There are two approaches to construct a small-world model: one is to reconnect the nodes with a probability in the nearest-neighbour coupled network which is proposed by Watts and Strogatz [27]; the other is to add new edges to the nearest-neighbour coupled network which is proposed by Newman and Watts [28] subsequently. The nearest-neighbour coupled network here means the networks in which all the nodes form a ring and each node is linked to its $2 k$ nearest neighbours.
The models constructed by the two approaches are usually called Watts-Strogatz (WS) and Newman-Watts (NW) small-world networks, respectively, and the former one is used more frequently in building small-world networks. Specifically, it is built starting from an ordered lattice with a moderately high connectivity, which insures a high clustering of the network. Then, each edge is removed with probability $p$ and reconnected between two randomly chosen nodes. This process creates a shortcut between two distant regions of the original network and makes the whole network compacter than before. The probability $p$ measures the degree of disorder or randomness of the resulting graph. For $p=0$ the order is fully preserved, while for $p=1$ a random graph is obtained. However, the average connectivity of the network will not be changed because no other edges are added to the network during the constructing process.

Setting the group size $N=200$, half of the nearest neighbours linked to one node in the starting nearest-neighbour coupled network $k=2$, and the reconnecting probability $p=0.2$, we can get a small-world network with the topological structure represented in Fig. 2.

It is obvious that the mean degree of the smallworld network $\langle k\rangle$ is $4(=2 k)$ and the sum degree is $800(=2 N k)$. By analysing the statistical properties of this network we can find the average distance is 4.926 (with the distance-based cohesion $=0.236$ ) which means that 4.926 steps are needed if one agent wants to connect to any one of the network.

In order to understand the structure of the network, it is necessary to measure the centrality of the network which is an important content in complex network researches. Three indexes are used frequently in relevant researches: degree centrality, closeness centrality, and betweenness centrality [29]. Historically, the first and conceptually the simplest is degree centrality, which is defined as the number of links incident upon a node; closeness centrality is defined as the total graph-theoretic distance to all other nodes in the network; and betweenness centrality is defined as the number of geodesic paths that pass through a node. The three indexes can also be used to measure the centrality of a graph via some transition, and they can be calculated by the following three formulas: 

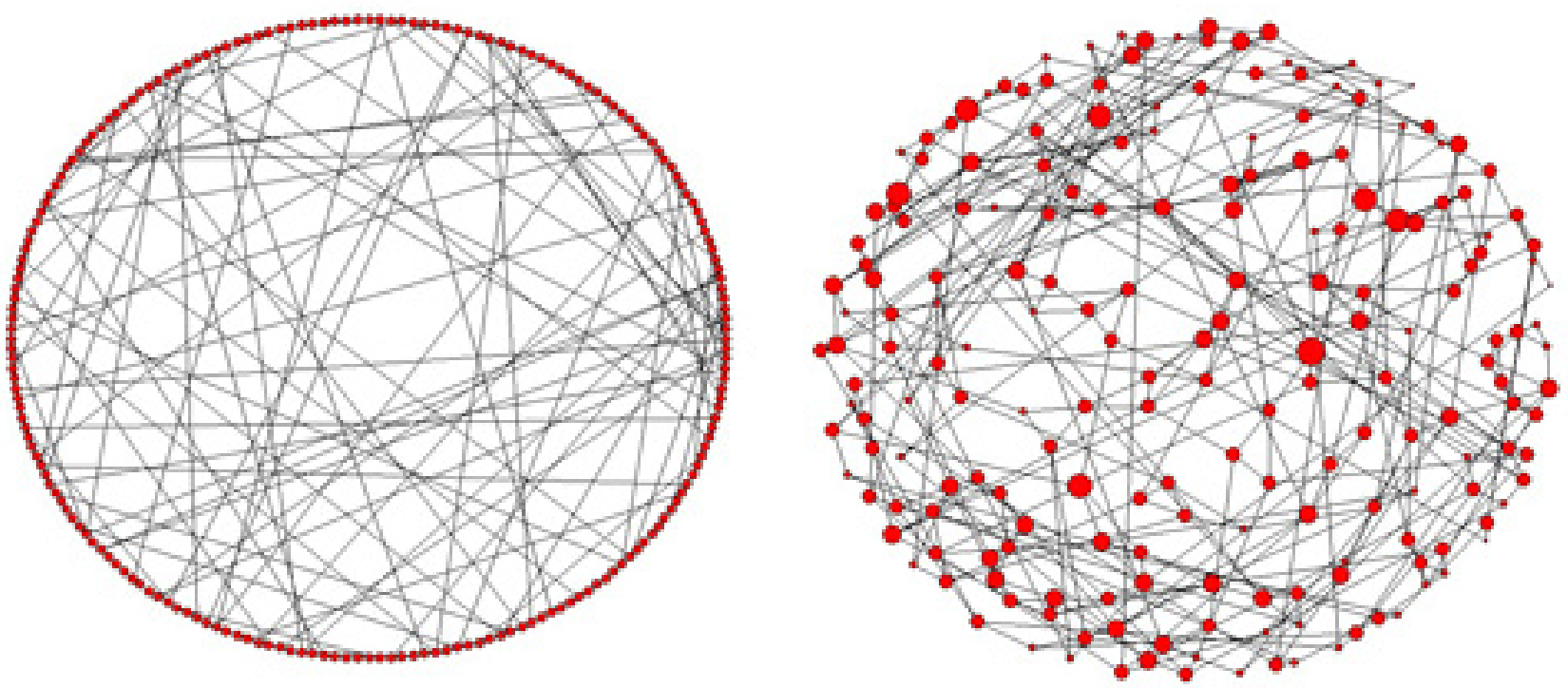

Fig. 2. Topological graph of our constructed small-world network. The left figure shows a circular form of the structure with all the nodes in a ring, and the size of the nodes serves no real purpose. The right figure shows another form of the network structure in which the size of the node reflects the magnitude of its node degree (the number of its direct connections to other nodes). With a big node comes a big degree.

$C_{\mathrm{D}}=\frac{\sum_{i=1}^{n}\left[C_{\mathrm{D}}(v)-C_{\mathrm{D}}(i)\right]}{(n-1)(n-2)}$,

where $C_{\mathrm{D}}(i)=\operatorname{degree}(i)$,

$C_{\mathrm{C}}=\frac{\sum_{i=1}^{n}\left[C^{\prime}{ }_{\mathrm{C}}(v)-C^{\prime}{ }_{\mathrm{C}}(i)\right]}{(n-1)^{2}(n-2)}(2 n-3)$,

where $C_{C}^{\prime}(i)=\sum_{j=1}^{n} d_{i j}$,

$$
C_{\mathrm{B}}=\frac{\sum_{i=1}^{n}\left[C_{\mathrm{B}}(v)-C_{\mathrm{B}}(i)\right]}{(n-1)^{2}(n-2)},
$$

where $C_{\mathrm{B}}(i)=\sum^{n} \sum^{n} b_{j q}(i), j \neq q \neq i$ and $j<q$.

In the above formulas, $v$ is the node with the highest degree, closeness, and betweenness centrality, respectively. By calculating with UCINET we can get three values of the network: $C_{\mathrm{D}}=0.0152$, $C_{\mathrm{C}}=0.0673$, and $C_{\mathrm{B}}=0.0458$. Therefore, the centrality of the network is very weak. In other words, the network does not have a significant trend of centralizing, and the nodes in the network are decentralized. In addition, the overall graph clustering coefficient is 0.254 , which declares that the clustering characteristic is good in this network. According to the rules set in Section 2, we can get the simulation results of opinions dynamics as shown in Fig. 3.

As is shown in Fig. 3, the time evolution of opinions starts from a uniform opinion distribution and ends up in gathering opinions into several clusters which are separated and which the agents in different clusters will not exchange anymore. Because the above five processes are all based on the data with the same initial opinion profile and the same distributed $\mu$, the results could well reflect the influence of the threshold $\varepsilon$ on the opinion dynamics. By comparing the evolution processes of opinions with different $\varepsilon$, we can find that the threshold $\varepsilon$ has a major impact on the final number of opinion clusters. It is easy to imagine that a higher value of $\varepsilon$ always indicates a higher tolerance to other people's opinions. Therefore, individuals are more likely to interact with others, and the group is more likely to reach a consensus. Weisbuch and other scholars have previously argued that the final number of opinion clusters varies with the integer part of $1 / 2 \varepsilon$, which is usually called " $1 / 2 \varepsilon$ rule" [3]. According to our simulation results, we have found that the rules do not apply here. In fact, the final number is always 
(a)

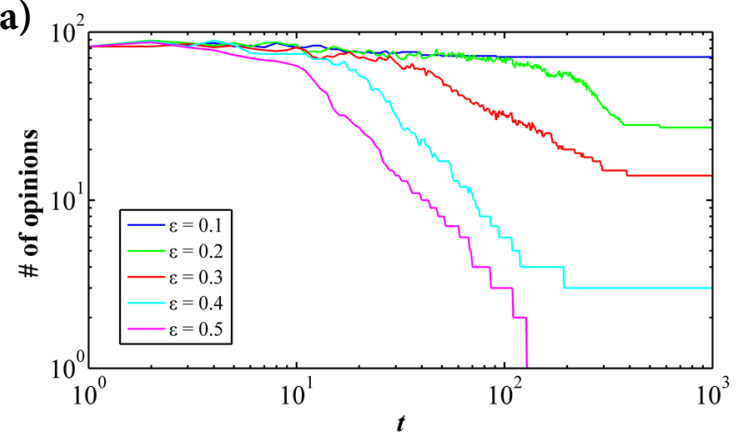

(c)

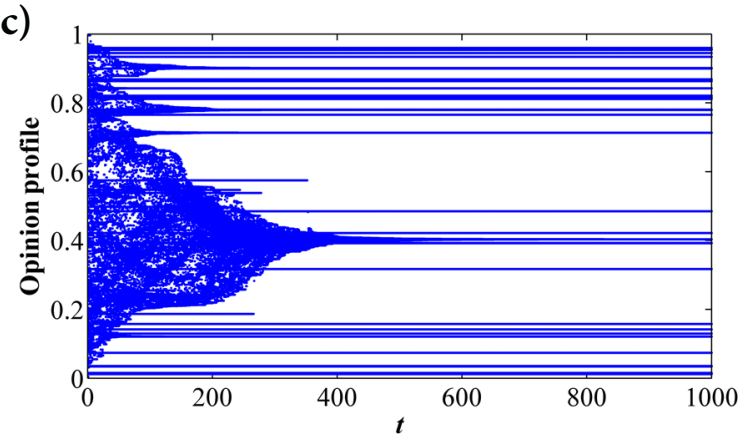

(e)

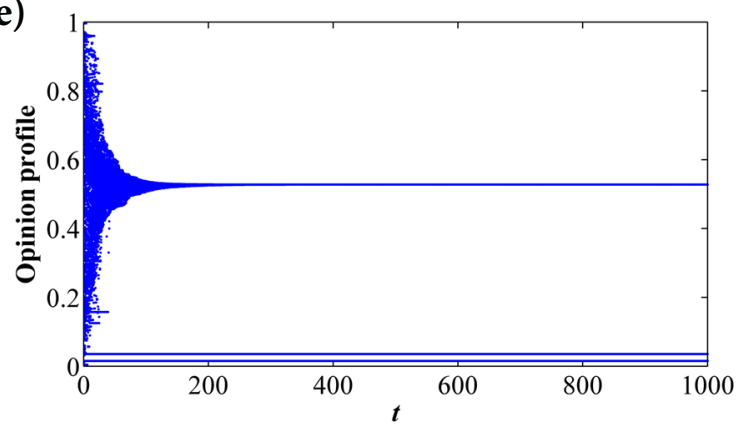

(b)

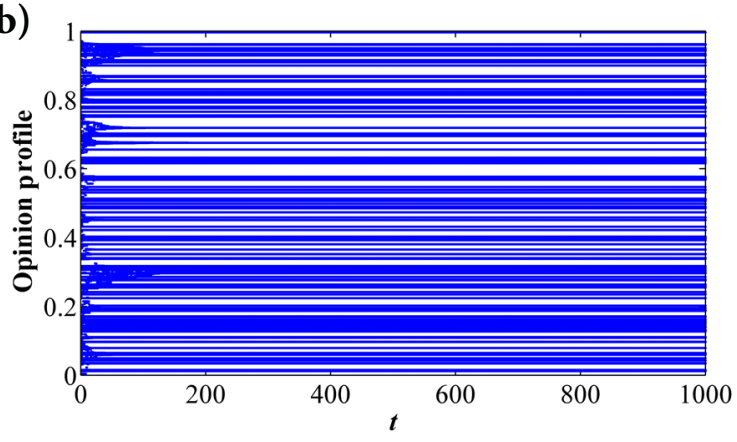

(d)

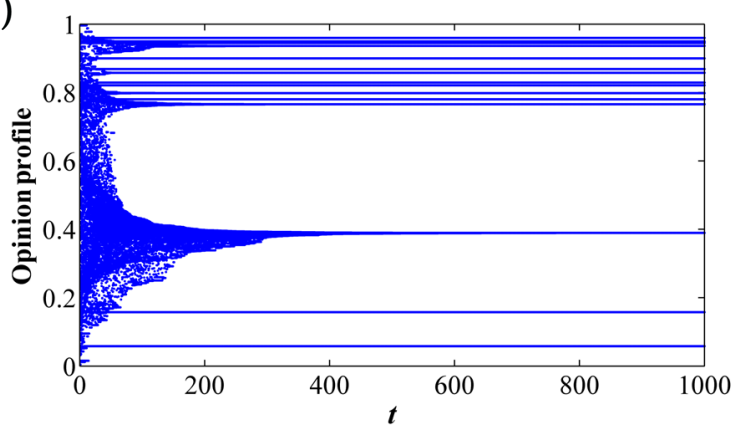

(f)

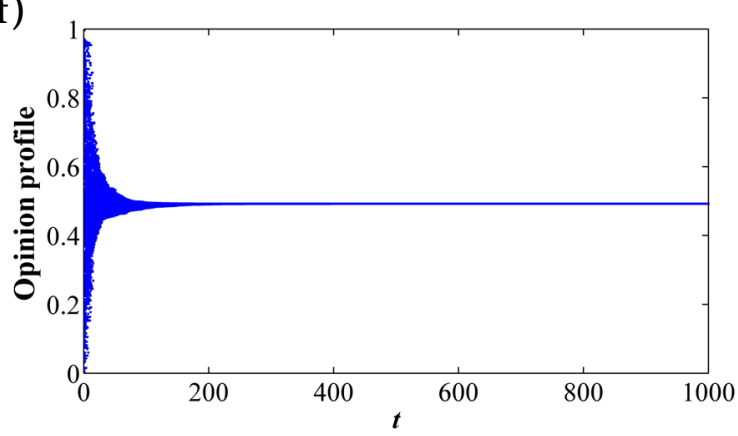

Fig. 3. Opinion dynamics in our constructed small-world network: (a) represents variation of the number of opinions held by all the agents, (b)-(f) describe opinion evolution with a given threshold $\varepsilon=0.1,0.2,0.3,0.4$, and 0.5 .

bigger than $1 / 2 \varepsilon$ in those cases in which individuals are connected as a social network with a certain structure in which one individual cannot exchange his opinion with anyone directly unless there is an edge between them. The result of consensus is hard to emerge, especially when the scale of the group is large, and a much more common case is that most agents are converging into a few opinion clusters. It has become a common cognition that people in the society are connected as a network in which they themselves are the nodes and their connections are the edges, and information is spreading through the path which is formed by chains of edges. Ignoring this fact in the modelling process will probably give rise to some inaccurate results. Opinion dynamics models are mostly agent-based models, so it is necessary to take the network structure into account.

\subsection{Opinion dynamics in BA network}

A large amount of work on the characterization of the topological properties of real networks has motivated the need to construct graphs with power law degree distributions [30]. There are many examples of real networks in which the structural changes are ruled by the dynamical evolution of the system. The BA scale-free model is a typical one of this class whose primary goal is to reproduce the growth processes taking place in real networks, and it is based on two basic ingredients: growth and preferential attachment [31]. The former indicates that networks expand continuously by addition of new nodes, and the latter means that new nodes attach preferentially to the sites already well connected. More precisely, an undirected graph $G_{\mathrm{BA}}(N, K)$ can 
be constructed as follows. Starting with $m_{0}$ nodes (the number of links among them is $k^{\prime}$ ), at each time-step $t=1,2,3, \ldots, N-m_{0}$ a new node $j$ with $m \leq m_{0}$ links is added to the network. The probability that a link will connect $j$ to an existing node $i$ is linearly proportional to the actual degree of $i$ :

$$
\prod_{j \rightarrow i}=\frac{k_{i}}{\sum_{j} k_{j}} .
$$

Because every new node has $m$ edges, the network at time-step $t$ will have $N=m_{0}+t$ nodes and $K=k^{\prime}+m t$ edges, corresponding to an average degree $\langle k\rangle \approx 2 m$ for large time-steps and small initial nodes. Suppose the group scale $N=200$, the number of initial nodes $m_{0}=4$ which compose a globally coupled network, and $m=2$. We can get a BA scale-free network as follows:

By some simple calculation we can obtain that the mean degree of our constructed BA network is 3.910 and the sum degree is 782 , which are basically the same as in our constructed small-world network. The clustering characteristic is not obvious in this network because the overall graph clustering coefficient is very small $(C=0.106)$. But besides that, the properties of the two networks are very different. The average distance of the BA network is 3.355 (with the distance-based cohesion $=0.327$ )

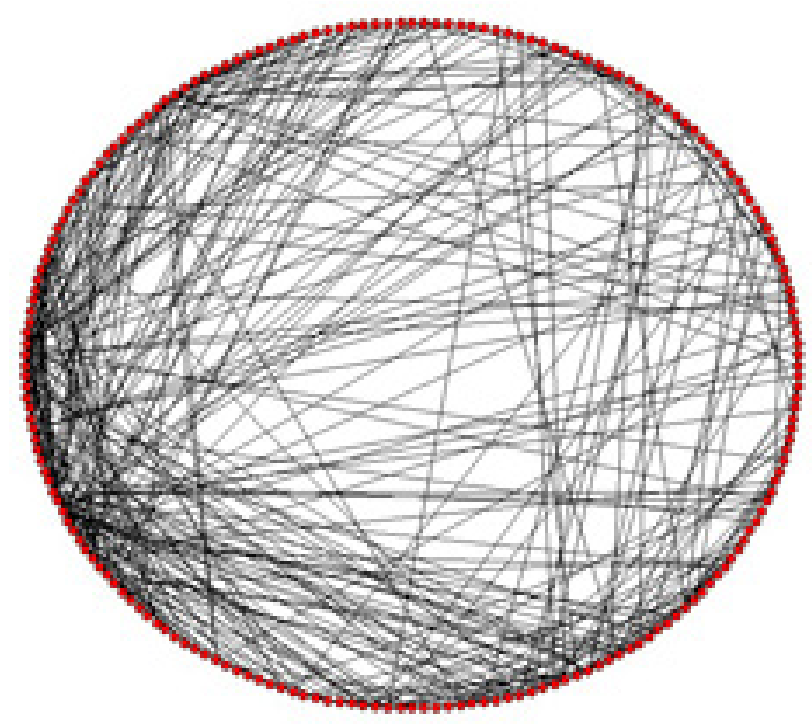

which is smaller than 4.926. This indicates that one agent could connect with any one of the network via 3.355 steps, and agents are more likely to establish relations with each other. From the three centrality indexes of the network (degree centrality $C_{\mathrm{D}}=0.1375$, closeness centrality $C_{\mathrm{C}}=0.3102$, and betweenness centrality $C_{\mathrm{B}}=0.2275$ ), we can find that the BA network has a more obvious centrality, which means that some nodes of the network have the power to attract other nodes to connect with them. As can be seen from the left topological graph in Fig. 4, the edges on the left side of the doughnut are much more intensive than those on the right side. The right graph also indicates that several nodes are much bigger than others as there are many more edges connecting them with other nodes. They reveal a major property of power-law networks: a small number of nodes have a large number of links. These nodes can be called rich nodes [19]. They tend to be tightly interconnected between themselves and form a rich-club [20]. The connectivity of the rich-club plays an important role in the functionality of the network, for example, in the transmission of rumours in social networks [32] or efficient delivery of information on the Internet [19]. The density of the connections between rich nodes can be quantified by the richclub coefficient:

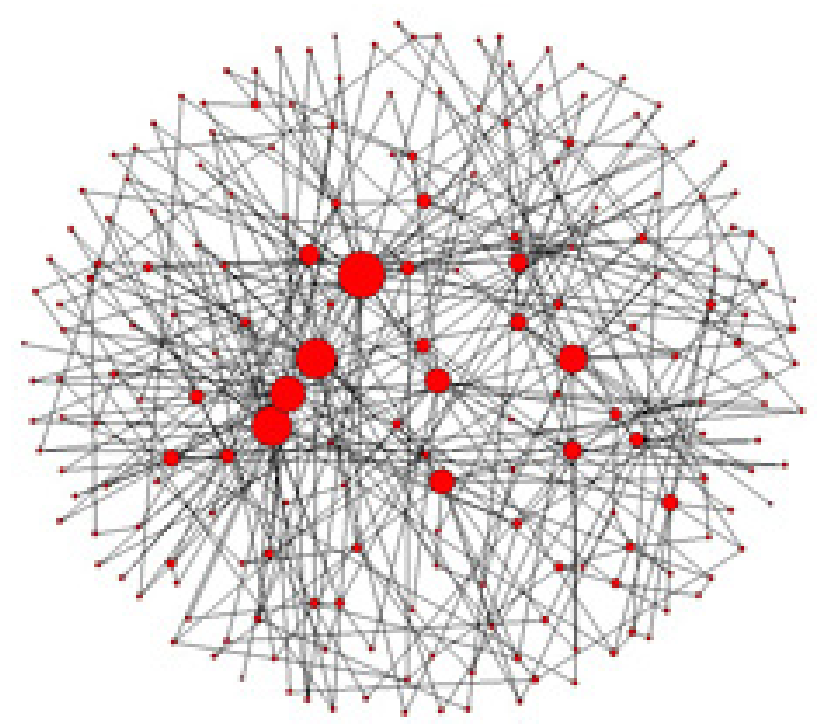

Fig. 4. Topological graph of the constructed BA network. Compared with our constructed small-world network, it seems that there are more links in the doughnut of the BA network, but in fact the links of the two networks are basically the same. The reason why we have the illusion is that most links are in the middle of the doughnut but on the edge of the ring. 


$$
\phi(r / N)=\frac{L}{r(r-1) / 2}=\frac{2 L}{r(r-1)},
$$

where $L$ denotes the number of edges among rich nodes, $r$ is the number of rich nodes, and $r(r-1) / 2$ represents the maximum possible number of edges among the rich nodes. According to Eq. (8), we can calculate the rich-club coefficient of the network $\varphi(r / N)=(2 \times 5) /(4 \times 3)=0.83$, which indicates that the rich-club connectivity is good. Based on this network, we can get the following results of opinion dynamics:

As can be seen from Fig. 5(a), opinion dynamics ends up in gathering opinions in some separated clusters, and as the threshold $\varepsilon$ is increasing from 0.1 to 0.5 , the number of final opinion clusters is

(a)

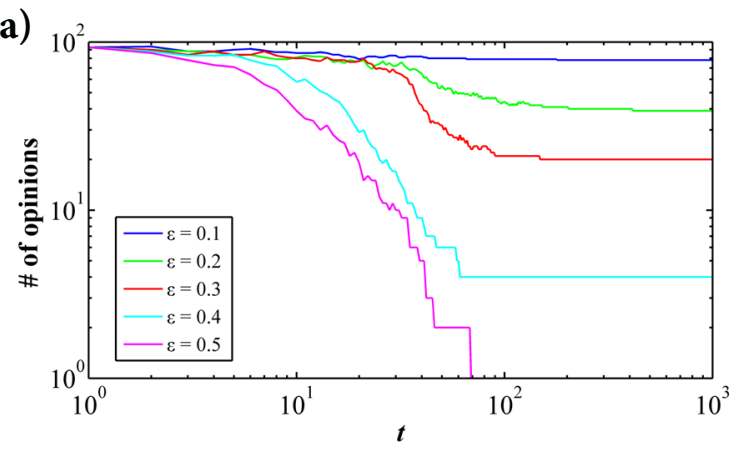

(c)

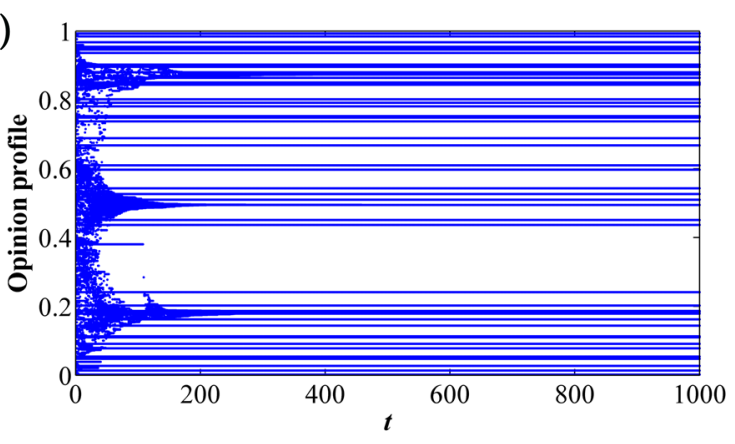

(e)

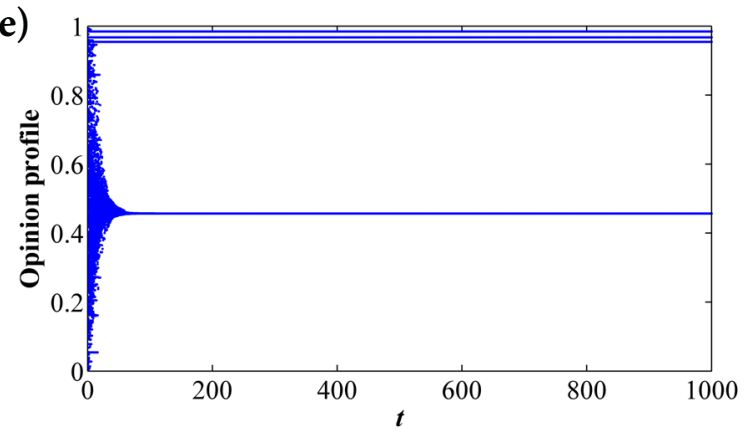

decreasing obviously. Figure 5(b-f) details the processes of opinion dynamics with $\varepsilon=0.1,0.2,0.3,0.4$, and 0.5 in the constructed BA network. From the simulation results we can find that there are no significant differences on the number of final opinion clusters between our constructed BA Network and small-world network. It indicates that the structure of the networks will have little or even no influence on the final number of opinion clusters in the case that the number of nodes and edges are basically the same. Therefore, we can draw the conclusion that the final number of opinion clusters depends on the value of bounded confidence in our models as it does in the WD model. However, compared with the evolution speed in the small-world network, the opinions of agents are converging at a much faster rate when dynamics happen in the BA network. The
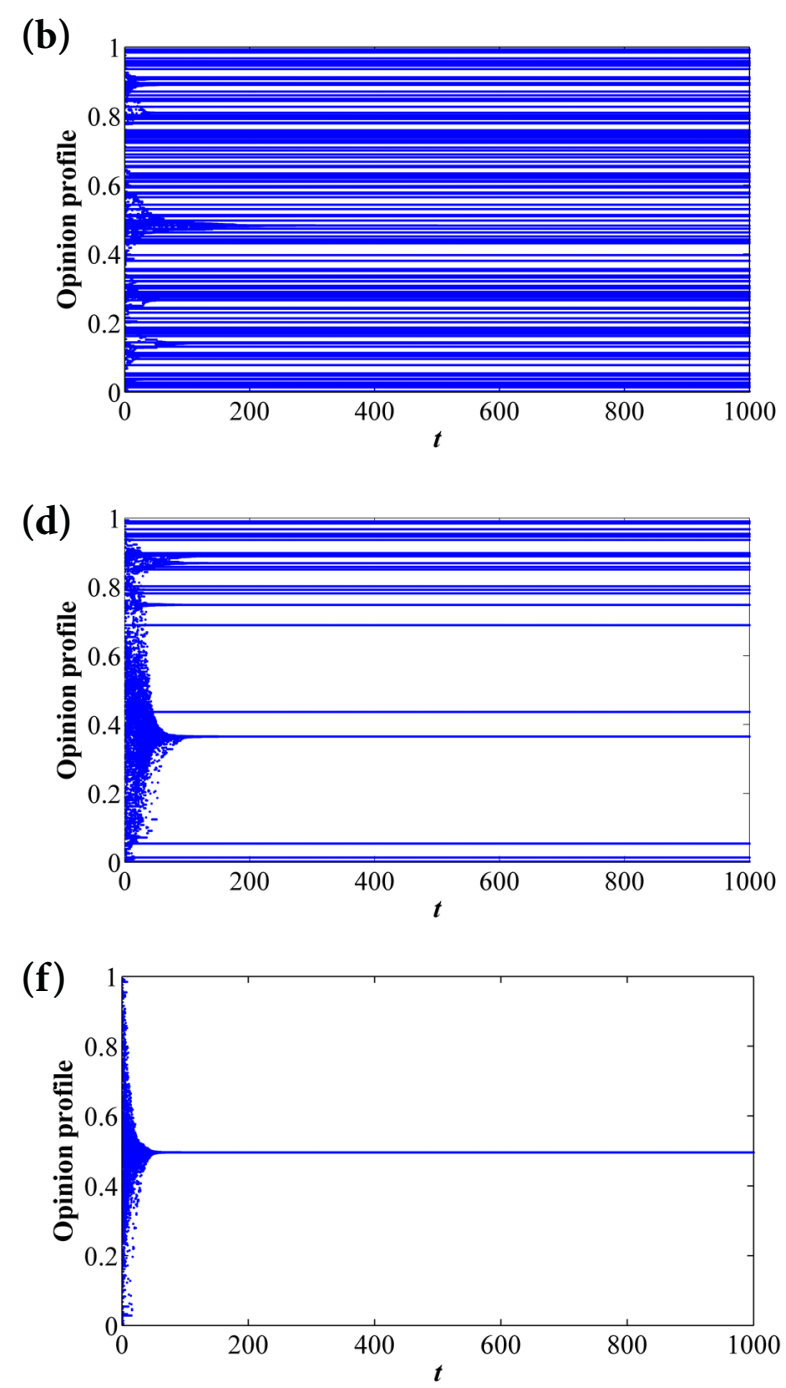

Fig. 5. Opinion dynamics in our constructed BA network: (a) represents variation of the number of opinions held by all the agents, (b)-(f) describe opinion evolution with $\varepsilon=0.1,0.2,0.3,0.4$, and 0.5 . 
evolution processes are all tending to be stable at the time-step $t \approx 200$ (in our constructed small-world network, the time-step $t \approx 400$ ); when $\varepsilon=0.4$ and 0.5 , the time-step that the evolution needed is much fewer (about 70 time-steps; in Fig. 3, the number is about 120 and 200). It happens because the centrality of the network can be strengthened by the rich nodes (they represent the special individuals that we can call opinion leaders) and their tight connection, which is shown clearly by the three centrality indexes of our constructed BA network. In fact, the ego network of the four rich nodes whose degrees are all more than 20 in this BA network has covered 42 per cent of the total 200 nodes. The importance of these rich nodes for the spreading of information and the forming of consensus has been proved by the twostep flow of communication and multistep flow theory which was first introduced by a sociologist Paul Lazarsfeld et al. [33]. According to their theory, people are all vertexes of a complex social network, and most people form their opinions under the influence of opinion leaders, who in turn are influenced by the mass media. It is because of the influence of opinion leaders that the structure cohesion [34] of the network is well strengthened, and the structure cohesion is one of the most important properties of the BA network what distinguishes it from the normal small-world network. Therefore, we can conclude that the existence of rich nodes (opinion leaders) and their tight connection in the BA network could accelerate the converging rate of people's opinions significantly.

\section{Conclusions}

Many agent-based models which are used to simulate the formulation of opinion in a social system have been proposed in modern sociophysics researches. In these models, including discrete and continuous models, the key feature is the interaction of individuals (agents). Generally, it is assumed that an individual is influenced by another one who is randomly chosen from the group. In this paper we propose a continuous opinion dynamics model by modifying the distribution of the convergence parameter $\mu$ in the WD model. We hold the view that the acceptance of others' opinions is not the same for different individuals. For this consideration we set up a precondition that the convergence parameter $\mu$ is not a fixed value but a series of values which obey a certain type of statistical distribution. In our model, opinion dynamics is dependent on the ego network of individuals which means that opinion exchange can only happen between two connected individuals. By comparing the opinion dynamics in our constructed WS small-world network and BA scale-free network, we can find that the final opinion clusters of dynamics are basically the same, but the converging rate is much faster when dynamics happens in the BA network. The simulation results prove that the bounded confidence is also the major factor which decides the final number of opinion clusters as in the WD model. Besides, compared with the small-world network, the rich nodes in the BA network take up most of the edges in the network which would bring about a higher centrality of the network. This property of the BA network gives an enormous impetus to push the opinions evolving at a much faster rate. It should be noted that the number of nodes and edges in our constructed networks remains the same during the process of opinion dynamics for convenience because we concentrate on the influence of the network structure on opinion dynamics in this paper. Further studies on opinion dynamics in an open network will be summarized in our following works.

\section{Acknowledgement}

We thank the referee for helpful suggestions and comments. This work is supported by the Humanity and Social Science Foundation of the Ministry of Education of China (Grant No. 12YJA840001) and the Major Program of the National Social Science Foundation of China (Grant No. 12\&ZD083).

\section{References}

[1] S. Galam and S. Moscovici, Towards a theory of collective phenomena: consensus and attitude changes in groups, Eur. J. Soc. Psycho. 21(1), 4974 (1991).

[2] K. Sznajd-Weron and J. Sznajd, Opinion evolution in closed community, Int. J. Mod. Phys. C 11(06), 1157-1165 (2000).

[3] G. Deffuant, D. Neau, F. Amblard, and G. Weisbuch, Mixing beliefs among interacting agents, Adv. Complex Syst. 3(01n04), 87-98 (2000).

[4] G. Weisbuch, G. Deffuant, F. Amblard, and J.- P. Nadal, Meet, discuss, and segregate!, Complexity 7(3), 55-63 (2002).

[5] G. Weisbuch, G. Deffuant, F. Amblard, and J.-P. Nadal, Interacting agents and continuous opinions 
dynamics, in: Heterogenous Agents, Interactions and Economic Performance (Springer, Berlin, 2003) pp. 225-242.

[6] R. Hegselmann and U. Krause, Opinion dynamics and bounded confidence models, analysis, and simulation, J. Artif. Soc. Soc. Simulat. 5(3), 1-33 (2002).

[7] J. Lorenz, Continuous opinion dynamics under bounded confidence: A survey, Int. J. Mod. Phys. C 18(12), 1819-1838 (2007).

[8] G.-S. Javier, G. Carl, and L.B. Jean-Yves, The bounded confidence model of opinion dynamics, Math. Model Meth. Appl. Sci. 22, 1150007 (2012).

[9] D. Stauffer and H. Meyer-Ortmanns, Simulation of consensus model of Deffuant et al. on a BarabasiAlbert network, Int. J. Mod. Phys. C 15(02), 241246 (2004).

[10]E. Kurmyshev, H.A. Juárez, and R.A. GonzálezSilva, Dynamics of bounded confidence opinion in heterogeneous social networks: concord against partial antagonism, Physica A 390(16), 2945-2955 (2011).

[11]B. Kozma and A. Barrat, Consensus formation on adaptive networks, Phys. Rev. E 77(1), 016102 (2008).

[12] B. Kozma and A. Barrat, Consensus formation on coevolving networks: groups' formation and structure, arXiv:0801.4860 (2008).

[13] P.-P. Li, D.-F. Zheng, and P. Hui, Dynamics of opinion formation in a small-world network, Phys. Rev. E 73(5), 056128 (2006).

[14]D. Jacobmeier, Multidimensional consensus model on a Barabási-Albert network, Int. J. Mod. Phys. C 16(04), 633-646 (2005).

[15]A. Sousa, Bounded confidence model on a still growing scale-free network, arXiv preprint condmat/0406766 (2004).

[16] W. Ru and C. Li-Ping, Opinion dynamics on complex networks with communities, Chin. Phys. Lett. 25(4), 1502-1505 (2008).

[17]Q. Li, L.A. Braunstein, H. Wang, J. Shao, H.E. Stanley, and S. Havlin, Non-consensus opinion models on complex networks, J. Stat. Phys. 151, 92-112 (2012).

[18] J.J. McAuley, L. da Fontoura Costa, and T.S. Caetano, Rich-club phenomenon across complex network hierarchies, Appl. Phys. Lett. 91(8), 084103 (2007).
[19]S. Zhou and R.J. Mondragón, The rich-club phenomenon in the Internet topology, IEEE Comm. Lett. 8(3), 180-182 (2004).

[20] R.J. Mondragón and S. Zhou, Random networks with given rich-club coefficient, Eur. Phys. J. B 85(9), 1-6 (2012).

[21]D. Stauffer, Sociophysics simulations, Comput. Sci. Eng. 5(3), 71-75 (2003).

[22] G. Weisbuch, G. Deffuant, and F. Amblard, Persuasion dynamics, Physica A 353, 555-575 (2005).

[23] M. Jalili, Social power and opinion formation in complex networks, Physica A 392(4), 959-966 (2012).

[24] L. Guo and X. Cai, Continuous opinion dynamics in complex networks, Comm. Comput. Phys. 5(5), 1045-1053 (2009).

[25]E.T. Jaynes, Probability Theory: The Logic of Science (Cambridge University Press, 2003).

[26]D.H. Zanette, Dynamics of rumor propagation on small-world networks, Phys. Rev. E 65(4), 041908 (2002).

[27]D.J. Watts and S.H. Strogatz, Collective dynamics of 'small-world' networks, Nature 393(6684), 440-442 (1998).

[28] M.E. Newman and D.J. Watts, Renormalization group analysis of the small-world network model, Phys. Lett. A 263(4), 341-346 (1999).

[29] L.C. Freeman, Centrality in social networks conceptual clarification, Soc. Network 1(3), 215-239 (1979).

[30] S. Boccaletti, V. Latora, Y. Moreno, M. Chavez, and D.-U. Hwang, Complex networks: Structure and dynamics, Phys. Rep. 424(4), 175-308 (2006).

[31] A.-L. Barabási and R. Albert, Emergence of scaling in random networks, Science 286(5439), 509-512 (1999).

[32]N. Masuda and N. Konno, Multi-state epidemic processes on complex networks, J. Theor. Biol. 243(1), 64-75 (2006).

[33] P.F. Lazarsfeld, B. Berelson, and H. Gaudet, The People's Choice: How the Voter Makes up His Mind in a Presidential Campaign (Duell, Sloan and Pearce, New York, 1944).

[34] P.V. Marsden and N.E. Friedkin, Network studies of social influence, Sociol. Meth. \& Res. 22(1), 127-151 (1993).

\title{
NUOMONIŲ DINAMIKA SUDE்TINGUOSE TINKLUOSE
}

\author{
Wei Zhang ${ }^{a}$, Ming-sheng $\mathrm{He}^{\mathrm{b}}$, Rui Jin ${ }^{\mathrm{b}}$ \\ ${ }^{a}$ Harbino technologijos universiteto Vadybos mokykla, Harbinas, Kinijos Liaudies Respublika \\ ${ }^{\mathrm{b}}$ Rytu Kinijos politikos mokslu ir teisés universiteto Socialinés pletros mokykla, Šanchajus, Kinijos Liaudies Respublika
}

